\title{
Dependences of the Casimir-Polder interaction between an atom and a cavity wall on atomic and material properties
}

\author{
V M Mostepanenko ${ }^{1}$, J F Babb ${ }^{2}$, A O Caride ${ }^{3}$, \\ G L Klimchitskaya ${ }^{4}$ and S I Zanette ${ }^{3}$ \\ ${ }^{1}$ Noncommercial Partnership "Scientific Instruments", Moscow, Russia \\ ${ }^{2}$ Institute for Theoretical Atomic, Molecular and Optical Physics, \\ Harvard-Smithsonian Center for Astrophysics, Cambridge, MA 02138, USA \\ ${ }^{3}$ Centro Brasileiro de Pesquisas Físicas, Rio de Janeiro, RJ 22290-180, Brazil \\ ${ }^{4}$ North-West Technical University, Millionnaya St. 5, St.Petersburg, Russia
}

\begin{abstract}
The Casimir-Polder and van der Waals interactions between an atom and a flat cavity wall are investigated under the influence of real conditions including the dynamic polarizability of the atom, actual conductivity of the wall material and nonzero temperature of the wall. The cases of different atoms near metal and dielectric walls are considered. It is shown that to obtain accurate results for the atomwall interaction at short separations, one should use the complete tabulated optical data for the complex refractive index of the wall material and the accurate dynamic polarizability of an atom. At relatively large separations in the case of a metal wall, one may use the plasma model dielectric function to describe the dielectric properties of wall material. The obtained results are important for the theoretical interpretation of experiments on quantum reflection and Bose-Einstein condensation.
\end{abstract}

PACS numbers: 34.50.Dy, 12.20.Ds, 34.20.Cf

Recently the study of dispersion interactions between an atom and a wall has assumed a new significance in connection with Bose-Einstein condensates of ultracold atoms [1-3]. The van der Waals and Casimir-Polder forces acting between dilute individual atoms, confined in a magnetic trap, and a wall may influence the stability of a condensate and the effective size of the trap [3]. As was shown in Ref. [4], the study of the collective oscillations of the Bose-Einstein condensate can provide a sensitive test of dispersion forces. This prediction was later supported both theoretically [5] and experimentally [6. Dispersion interaction between an atom and a wall is also taken into account in quantum reflection of cold atoms on a surface [7] and in dynamical interaction effects of fast atoms and molecules with solid surfaces $[8]$. Currently the new asymptotic behavior of the surface-atom interaction out of thermal equilibrium has been advanced [9]. Below we use the generic name "Casimir-Polder" for all atom-wall interactions of dispersion 
nature because the pure nonretarded regime occurs at separations from zero to a few nanometers only.

The theoretical basis for the description of the Casimir-Polder interaction between an atom at a separation $a$ from a flat wall at temperature $T$ in thermal equilibrium is given by the Lifshitz-type formula for the free energy [10-12]

$$
\begin{aligned}
\mathcal{F}(a, T) & =-\frac{k_{B} T}{8 a^{3}}\left\{2 \alpha(0) f(0)+\sum_{l=1}^{\infty} \alpha\left(i \zeta_{l} \omega_{c}\right)\right. \\
& \left.\times \int_{\zeta_{l}}^{\infty} d y e^{-y}\left[\left(2 y^{2}-\zeta_{l}^{2}\right) r_{\|}\left(\zeta_{l}, y\right)+\zeta_{l}^{2} r_{\perp}\left(\zeta_{l}, y\right)\right]\right\} .
\end{aligned}
$$

Here $\alpha(\omega)$ is the atomic dynamic polarizability, $k_{B}$ is the Boltzmann constant, $\zeta_{l}=4 \pi l k_{B} T a /(\hbar c)$ are the dimensionless Matsubara frequencies, $\omega_{c}=c /(2 a)$ is the characteristic frequency of the Casimir-Polder interaction, and the reflection coefficients for two independent polarizations of electromagnetic field are defined as

$$
r_{\|}\left(\zeta_{l}, y\right)=\frac{\varepsilon_{l} y-\sqrt{y^{2}+\zeta_{l}^{2}\left(\varepsilon_{l}-1\right)}}{\varepsilon_{l} y+\sqrt{y^{2}+\zeta_{l}^{2}\left(\varepsilon_{l}-1\right)}}, \quad r_{\perp}\left(\zeta_{l}, y\right)=\frac{\sqrt{y^{2}+\zeta_{l}^{2}\left(\varepsilon_{l}-1\right)}-y}{\sqrt{y^{2}+\zeta_{l}^{2}\left(\varepsilon_{l}-1\right)}+y},
$$

where $\varepsilon_{l} \equiv \varepsilon\left(i \zeta_{l} \omega_{c}\right)$ is the permittivity of wall material computed at imaginary Matsubara frequencies. For dielectrics $f(0)=[\varepsilon(0)-1] /[\varepsilon(0)+1]$ and for metals $f(0)=1$.

In most calculations of the atom-wall interaction previously performed only the limiting cases of large and short separations were considered. The polarizability of the atom was taken into account in the static approximation [13] or in the framework of the single-oscillator model [14], and the dielectric properties of the wall material were oversimplified (for example, by considering a metal wall to be made of ideal metal). The present experimental situation requires precise (1\% accuracy) computations of the Casimir-Polder interaction in a wide separation range from about $3 \mathrm{~nm}$ (where the Lifshitz formula becomes applicable) to $10 \mu \mathrm{m}$. In this paper we present the results of such computations clarifying the atomic and material properties which are essential to attain the required accuracy.

We have performed numerical computations of the free-energy (11), (2) for metastable $\mathrm{He}^{*}, \mathrm{Na}$, and $\mathrm{Cs}$ atoms in ground state located near metal (Au), semiconductor $(\mathrm{Si})$ and dielectric $\left(\mathrm{SiO}_{2}\right)$ walls at $T=300 \mathrm{~K}$. (The modification on account of walls in the spontaneous emission of Rydberg atoms, obtained, e.g., by means of two lasers, is discussed in Refs. [15, 16]. However, thermal quanta at $T=300 \mathrm{~K}$ are too small to excite atom from the ground state to some other states.) Three different descriptions for the dielectric properties of a metal were used: $i$ ) as an ideal metal, $i i$ ) using the dielectric permittivity from the free-electron plasma model $\varepsilon(i \xi)=1+\omega_{p}^{2} / \xi^{2}$ (where $\omega_{p}$ is the plasma frequency), and $\left.i i i\right)$ with $\varepsilon(i \xi)$ obtained by means of dispersion relation using the tabulated optical data for the complex index of refraction [17. The dielectric permittivity of a semiconductor or dielectric was described either by their static permittivity $\varepsilon(0)$ or by means of their tabulated optical data and the dispersion 
Table 1. Free energy $\mathcal{F}$ (in $J$ ) of the Casimir-Polder interaction between a $\mathrm{He}^{*}$ atom and $\mathrm{Au}$ and $\mathrm{SiO}_{2}$ walls [columns (a)] and correction factors to it at different separations $a$. In columns labeled (a) the material of the wall and the atom are described by the optical tabulated data and accurate dynamic polarizability, respectively. In columns labeled (b) the metal is an ideal one and the dielectric permittivity of $\mathrm{SiO}_{2}$ is static; the dynamic polarizability of the atom is the accurate one. In columns labeled (c) the wall materials are described by the tabulated optical data and the dynamic polarizability of the atom is given by the single-oscillator model. In column (d) the metal is described by the plasma model and the dynamic polarizability of the atom is accurate.

\begin{tabular}{cccccccccc}
\hline & \multicolumn{3}{c}{$\mathrm{He}^{*}$ near a Au wall } & & \multicolumn{3}{c}{$\mathrm{He}^{*}$ near a $\mathrm{SiO}_{2}$ wall } \\
\cline { 2 - 4 } \cline { 8 - 10 }$a(\mathrm{~nm})$ & $(\mathrm{a})$ & $(\mathrm{b})$ & $(\mathrm{c})$ & $(\mathrm{d})$ & & $(\mathrm{a})$ & $(\mathrm{b})$ & $(\mathrm{c})$ \\
\hline 3 & $3.80 \times 10^{-23}$ & 1.16 & 0.956 & 0.937 & & $1.61 \times 10^{-23}$ & 1.78 & 0.949 \\
10 & $9.95 \times 10^{-25}$ & 1.14 & 0.961 & 0.948 & & $4.18 \times 10^{-25}$ & 1.73 & 0.958 \\
20 & $1.18 \times 10^{-25}$ & 1.14 & 0.973 & 0.959 & & $4.94 \times 10^{-26}$ & 1.68 & 0.967 \\
50 & $6.62 \times 10^{-27}$ & 1.13 & 0.984 & 0.976 & & $2.71 \times 10^{-27}$ & 1.64 & 0.983 \\
100 & $6.98 \times 10^{-28}$ & 1.11 & 0.991 & 0.981 & & $2.76 \times 10^{-28}$ & 1.60 & 0.993 \\
150 & $1.77 \times 10^{-28}$ & 1.10 & 0.997 & 0.992 & & $6.93 \times 10^{-29}$ & 1.57 & 0.994 \\
\hline
\end{tabular}

relation. The polarizability of an atom was represented by its static value $\alpha(0)$ or by means of the highly accurate $N$-oscillator model [18]

$$
\alpha\left(i \zeta_{l} \omega_{c}\right)=\frac{e^{2}}{m} \sum_{n=1}^{N} \frac{f_{0 n}}{\omega_{0 n}^{2}+\omega_{c}^{2} \zeta_{l}^{2}},
$$

where $m$ and $e$ are the electron mass and charge, $f_{0 n}$ and $\omega_{0 n}$ are the oscillator strength and frequency of the $n$th excited-state to ground-state transition, respectively. A more simplified single-oscillator model [Eq. (3) with $N=1$ ] was also used.

Computations show that at short separations (from $3 \mathrm{~nm}$ to about $150 \mathrm{~nm}$ ) it is necessary to use the complete tabulated optical data for the complex index of refraction in order to find the most accurate results. For the dynamic polarizability of an atom, at shortest separations the highly accurate data for it should be used. With increasing atom-wall distance up to several tens of nanometers the single-oscillator model becomes applicable. These calculations are illustrated in Table 1 by the example of a metastable He* atom near $\mathrm{Au}$ and $\mathrm{SiO}_{2}$ walls (the analogous results for $\mathrm{Na}$ and $\mathrm{Cs}$ atoms near $\mathrm{Au}$, $\mathrm{Si}$, and $\mathrm{SiO}_{2}$ walls can be found in Refs. [11, 12]). The tabulated optical data for Au and $\mathrm{SiO}_{2}$ were taken from Ref. [19], and the values of $\mathrm{Au}$ plasma frequency and $\mathrm{SiO}_{2}$ static permittivity are $\omega_{p}=9.0 \mathrm{eV}=1.37 \times 10^{16} \mathrm{rad} / \mathrm{s}$ and $\varepsilon(0)=3.84$. The accurate data for the dynamic polarizability of metastable $\mathrm{He}^{*}$ (with a relative error of order $10^{-6}$ ) were taken from Ref. [20] and the parameters of a single-oscillator model from Ref. [21] were used. As is seen in Table 1, the use of the ideal metal or the static dielectric permittivity approximations leads to errors up to $16 \%$ for metal and $78 \%$ for dielectric. These errors slowly decrease with increasing separation between the atom and the wall. The plasma model is a better approximation than the ideal metal approximation. It results in errors of about $5 \%$ at the shortest separations and becomes sufficiently exact 


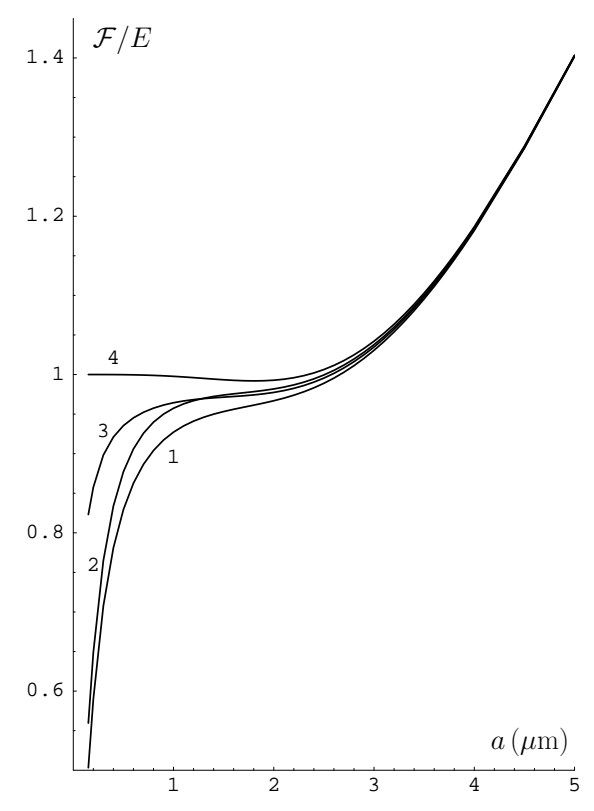

Figure 1. Normalized Casimir-Polder free energy for metastable $\mathrm{He}^{*}$ atom near $\mathrm{Au}$ wall versus separation. Lines 1,2 take into account the dynamic polarizability of an atom (in the single-oscillator model) and describe metal by the plasma model or as ideal one, respectively. Lines 3, 4 describe atom by the static polarizability and metal in analogy with lines 1,2 .

when the separation approaches $150 \mathrm{~nm}$. The use of the static atomic polarizability would result in much greater errors and for this reason it is omitted from Table 1.

At large separations, from $150 \mathrm{~nm}$ to a few micrometers, the effects of the atomic dynamic polarizability play a more important role than the effects of the finite conductivity of the metal. The single-oscillator model, however, is sufficient to achieve the required accuracy. The dielectric properties of a metal can be approximated by the plasma model. For dielectrics and semiconductors both tabulated optical data and the Ninham-Parsegian representation for the dielectric permittivity 22] are suitable for obtaining accurate results. For sufficiently large separations one can use the static dielectric permittivity of the wall. We illustrate these features using the example of a $\mathrm{He}^{*}$ atom near an $\mathrm{Au}$ wall. Due to the strongly nonmonotonous dependence of the free energy on separation, we plot along the vertical axis the ratio of the free energy to the Casimir-Polder energy $E(a)=-3 \hbar c \alpha(0) /\left(8 \pi a^{4}\right)$ of an atom near a wall made of ideal metal at $T=0$. As is seen from Fig. 1 , at separations $a>(4-5) \mu \mathrm{m}$ all approaches lead to approximately equal values of the free energy.

To conclude, results such as those presented in the columns labeled (a) in Table 1 and by line 1 in Fig. 1 can be used in interpretation of precision experiments on atomsurface interactions. 


\section{Acknowledgments}

ITAMP is supported in part by a grant from the NSF to the Smithsonian Institution and Harvard University. VMM and GLK were partially supported by FAPERJ (proceess Nos. E-26/170.132 and 170.409/2004) and by the Russian Foundation for Basic Research (grant No. 05-08-18119a).

\section{References}

[1] Harber D M, McGuirk J M, Obrecht J M and Cornell E A 2003 J. Low Temp. Phys. 133229

[2] Leanhardt A E, Shin Y, Chikkatur A P, Kielpinski D, Ketterle W and Pritchard D E 2003 Phys. Rev. Lett. 90100404

[3] Lin Y, Teper I, Chin C and Vuletić V 2004 Phys. Rev. Lett. 92050404

[4] Antezza M, Pitaevskii L P and Stringari S 2004 Phys. Rev. A 70053619

[5] Carusotto I, Pitaevskii L P, Stringari S, Modugno G and Inguscio M 2005 Phys. Rev. Lett. 95 093202

[6] Harber D M, Obrecht J M, McGuirk J M and Cornell E A 2005 Phys. Rev. A 72033610

[7] Oberst H, Tashiro Y, Shimizu K and Shimizu F 2005 Phys. Rev. A 71052901

[8] Villó-Pérez I, Abril I, Garcia-Molina R and Arista N R 2005 Phys. Rev. A 71052902

[9] Antezza M, Pitaevskii L P and Stringari S 2005 Phys. Rev. Lett. 95113202

[10] Lifshitz E M and Pitaevskii L P 1980 Statistical Physics, Part. II (Oxford: Pergamon Press)

[11] Babb J F, Klimchitskaya G L and Mostepanenko V M 2004 Phys. Rev. A 70042901

[12] Caride A O, Klimchitskaya G L, Mostepanenko V M and Zanette S I 2005 Phys. Rev. A 71042901

[13] Casimir H B G and Polder D 1948 Phys. Rev. 73360

[14] Boström M and Sernelius B E 2000 Phys. Rev. A 61052703

[15] Hinds E A, Lai K S and Schnell M 1997 Phil. Trans. R. Soc. Lond. A 3552353

[16] Wu S-T and Eberlein C 2000 Proc. R. Soc. Lond. A 4561931

[17] Bordag M, Mohideen U and Mostepanenko V M 2001 Phys. Rep. 3531

[18] Shih A and Parsegian V A 1975 Phys. Rev. A 12835

[19] Handbook of Optical Constants of Solids, ed. Palik E D 1985 (New York: Academic Press)

[20] Yan Z-C and Babb J F 1998 Phys. Rev. A 581247

[21] Brühl R, Fouquet P, Grisenti R E, Toennies J P, Hegerfeldt G C, Köhler T, Stoll M and Walter C 2002 Europhys. Lett. 59357

[22] Mahanty J and Ninham B W 1976 Dispersion Forces (New York: Academic Press) 